\title{
POLYNOMIAL SOLUTIONS OF PARTIAL DIFFERENTIAL EQUATIONS
}

\author{
W. K. Hayman and Z. G. Shanidze \\ Dedicated to Dick Askey on the occasion of his 65th birthday
}

\begin{abstract}
A method of finding polynomial solutions for partial differential equations with polynomial data is introduced. The solution of the problem for second order equations with given boundary values on a quadratic curve is discussed in detail, and examples are given to show the wide scope of the method.
\end{abstract}

\section{Introduction}

Conventionally, second order partial differential equations are divided into hyperbolic, elliptic, and parabolic equations according to the nature of the second order terms. The form of the data required to obtain a unique solution differ according to the type of equation. For instance, an elliptic equation such as that of Laplace has inside a Jordan curve $S$ a unique solution $u$, which assumes given continuous boundary values on $S$. For hyperbolic equations, such a result ceases to be true. The equation

$$
\frac{\partial^{2} u}{\partial x^{2}}-\frac{\partial^{2} u}{\partial y^{2}}=0
$$

has the solutions $u=0$ and $u=x^{2}+y^{2}-1$, both of which vanish on the circle $x^{2}+y^{2}=1$.

In this paper, we discuss the nature of polynomial solutions of such equations. In this case, it turns out that the difference between elliptic and hyperbolic equations largely disappears. Instead, the nature of the boundary curve becomes of crucial importance. For $n$ th-order equations, the curves must have roughly degree $n$ to give a unique solution with given arbitrary polynomial boundary values. If the curve or curves have smaller degree, there is, in general, a linear space of solutions. For boundary curves of higher degrees, solutions exist only for some special polynomial data.

An exceptional situation arises, for instance, when the boundary curve $S$ is part of the zero set of a polynomial solution of the equation (see above). In this case, uniqueness no longer occurs.

We shall start this paper by considering second order equations with constant coefficients. At the end of the paper, we shall give some examples to show that the technique can be extended to much more general situations.

The method was developed previously by the second author (see, eg., [3]). Exact polynomial solutions of this type first appeared in Mikeladze [2]. Somewhat similar ideas are contained in Abadi and Ortiz [1]. They used the method as a basis for

Received April 29, 1998, revised August 26, 1998.

1991 Mathematics Subject Classification: 35A05.

Key words and phrases: polynomial solutions, partial differential equations. 
numerical approximation. However, in this paper, we hope to introduce this general and flexible method to a wider readership.

\section{Examples of second order elliptic and hyperbolic equations}

Consider the equations

$$
u_{x x}-\varepsilon u_{y y}=0
$$

where $\varepsilon= \pm 1$.

Homogeneous $n$ th-degree solutions of (2.1) are

$$
r_{n}(x, y)=y^{n}+\varepsilon\left(\begin{array}{l}
n \\
2
\end{array}\right) x^{2} y^{n-2}+\varepsilon^{2}\left(\begin{array}{l}
n \\
4
\end{array}\right) x^{4} y^{n-4}+\cdots, \quad n=0,1,2, \ldots,
$$

and

$$
s_{n}(x, y)=n x y^{n-1}+\varepsilon\left(\begin{array}{l}
n \\
3
\end{array}\right) x^{3} y^{n-3}+\cdots .
$$

In both cases, $\varepsilon= \pm 1$, there are exactly $2 n+1$ linearly independent polynomial solutions of degree not exceeding $n$. Thus, we could expect a unique polynomial solution of degree up to $N$ if the coefficients of the polynomial

$$
u(x, y)=a_{0}+\sum_{1}^{N}\left\{a_{n} r_{n}(x, y)+b_{n} s_{n}(x, y)\right\}
$$

have to satisfy $2 N+1$ linear equations.

Consider the conic

$$
S: \quad x^{2}-\eta y^{2}=1 \text { where } \eta= \pm 1,
$$

and suppose that $u(x, y)$ satisfies (2.1) and, in addition,

$$
u(x, y)=P_{N}(x, y)
$$

on (2.3) where $P_{N}$ is a polynomial of degree $N$ in $x, y$. By means of (2.3), we can express $y^{2}$ in terms of $x^{2}$ and thus polynomials in $y^{2}$ in terms of polynomials in $x^{2}$. Hence, the most general polynomial of degree up to $N$ on (2.3) can be written in the form

$$
Q_{N}(x, y)=\sum_{0}^{N} a_{n} x^{n}+\sum_{0}^{N-1} b_{n} x^{n-1} y
$$

Thus, polynomial data of degree $N$ in $(x, y)$ can always be written in the form

$$
u(x, y)=Q_{N}(x, y) \text { on } S \text {. }
$$

A polynomial solution $u(x, y)$ of $(2.1)$ having degree $N$ takes the form

$$
u(x, y)=c_{0}+\sum_{1}^{N}\left\{c_{n} r_{n}(x, y)+c_{n}^{\prime} s_{n}(x, y)\right\} .
$$

On the curve $S$, we replace higher powers of $y^{2}$ by $1, y$ and so obtain a modified polynomial of the form (2.4) with coefficients that are linear combinations of the $c_{n}$, $c_{n}^{\prime}$. To make sure that $u(x, y)=Q_{N}(x, y)$ on $S$, we thus obtain $2 N+1$ linear equations for the $2 N+1$ coefficients $c_{n}, c_{n}^{\prime}$. These equations have a unique solution for arbitrary $a_{n}$ and $b_{n}$ if, and only if, the equations, when all the $a_{n}$ and $b_{n}$ vanish, have only the zero solution i.e., if the only solution of (2.1), which is a polynomial $P$ of degree at 
most $N$ and which vanishes on $S$, is identically zero. Thus, uniqueness may depend on the degree $N$ of the polynomial.

Example 1. Consider a solution such that $u=1+y$ on $S$.

If we allow only linear polynomials then, $u=1+y$ is the only solution. The same remains true for general polynomial solutions for the equation (2.1) if $\varepsilon=\eta=-1$. For, by the maximum principle, a harmonic polynomial vanishing on (2.3) vanishes identically in this case.

Consider next the case $\varepsilon=1, \eta=-1$. In this case, the polynomial $x^{2}+y^{2}-1$ satisfies (2.1) and vanishes identically on (2.3). Thus, in this case, if $N \geq 2$, there exist infinitely many or no polynomial solutions of (2.1) of degree $N$ which assume given polynomial data on (2.3). For if $u$ is such a solution, so is $u+a\left(x^{2}+y^{2}-1\right)$ for every real constant $a$. We see, for instance, that quadratic data on $S$ take the form

$$
P(x, y)=A x^{2}+B y^{2}+2 H x y+2 G x+2 F y+C .
$$

Substituting $x^{2}=1-y^{2}$, we can write this as

$$
(B-A) y^{2}+2 H x y+2 G x+2 F y+A+C .
$$

A polynomial of second degree satisfying (2.1) takes the form

$$
a\left(x^{2}+y^{2}\right)+2 h x y+2 g x+2 f y+c .
$$

Substituting $x^{2}=1-y^{2}$ on $S$ again, we obtain

$$
2 h x y+2 g x+2 f y+c+a .
$$

We see that, to make (2.7) and (2.8) identical, we need $A=B$. So our basic problem has infinitely many solutions if $A=B$ and none otherwise.

The situation is similar if $\varepsilon=-1, \eta=1$. In this case, $P=x^{2}-y^{2}-1$ vanishes on $S$ but not identically. There is no quadratic polynomial satisfying (2.1) and assuming values (2.6) on $S$ unless $A=-B$, and, in this case, there are infinitely many such polynomials.

Finally, we come to the case $\varepsilon=\eta=1$. In this case, we again have a unique quadratic solution for given quadratic data. We need only check that there is no quadratic polynomial $P(x, y)$ given by (2.6), which satisfies (2.1) and vanishes on (2.3), other than the zero polynomial. To see this, we recall that (2.1) implies $A=B$. If

$$
\Delta=A B-H^{2}>0,
$$

then $P$ has constant sign for large $x$ or $y$ and so cannot vanish on $S$. If $\Delta=0$ and $A=B=H=0$, then $P$ is linear and so vanishes at most at 2 points of $S$. Otherwise, suppose that $A>0$. Then

$$
P=(x \sqrt{A} \pm y \sqrt{B})^{2}+2 G x+2 F y+C .
$$

Hence, $P$ is large near at least one of the lines $y=x, y=-x$ and, so again, $P$ cannot vanish on $S$. If $A<0$, the conclusion is similar.

Finally, if $\Delta<0$ and $y= \pm(1+o(1)) x$, then we have

$$
P=(A+B-2 H)(1+o(1)) x^{2} .
$$


Further, since $A=B$ and $H^{2}>A^{2}$, we have $A+B-2 H \neq 0$. Thus again, $P$ cannot vanish identically on $S$.

Summing up, we see that our problem has a unique solution if $\varepsilon=\eta$, but not in general if $\varepsilon=-\eta$.

Example 2. We obtain the polynomial of degree 4, which satisfies Laplace's equation

$$
u_{x x}+u_{y y}=0
$$

and which on the ellipse or hyperbola

$$
S: \quad \frac{x^{2}}{a^{2}}+\varepsilon \frac{y^{2}}{b^{2}}=1, \quad \varepsilon= \pm 1
$$

assumes the polynomial values

$$
\left.u(x, y)\right|_{S}=c_{21} x^{2} y+c_{o 4} y^{4}, \quad c_{21}, c_{04} \in \mathbb{R} .
$$

Since the polynomial (2.11) has degree 4, we can by (2.2) express our solution in the form

$$
\begin{aligned}
u(x, y)=a_{o} & +a_{1} y+b_{1} x+a_{2}\left(x^{2}-y^{2}\right)+2 b_{2} x y \\
& +a_{3}\left(y^{3}-3 x^{2} y\right)+b_{3}\left(3 x y^{2}-y^{3}\right) \\
& +a_{4}\left(x^{4}-6 x^{2} y^{2}+y^{4}\right)+4 b_{4}\left(x^{3} y-x y^{3}\right)
\end{aligned}
$$

From (2.10), we obtain

$$
x^{2}=a^{2}\left(1-\varepsilon \frac{y^{2}}{b^{2}}\right)
$$

We substitute this in (2.11) and (2.12) to obtain a system of equations for the unknown coefficients. Assuming that

$$
b^{2}+a^{2} \neq 0, \quad b^{2}+3 \varepsilon a^{2} \neq 0, \quad \text { and } \quad a^{4}+6 \varepsilon a^{2} b^{2}+b^{4} \neq 0
$$

we obtain the unique solution

$$
\begin{aligned}
u(x, y)= & -\frac{a^{4} b^{4} c_{04}}{a^{4}+6 \varepsilon a^{2} b^{2}+b^{4}}+\left(a^{2} c_{21}-\frac{3 \varepsilon a^{4} c_{21}}{b^{2}+3 \varepsilon a^{2}}\right) y \\
& -\frac{2 a^{2} b^{4}\left(3 b^{2}+\varepsilon a^{2}\right)\left(x^{2}-y^{2}-a^{2}\right) c_{04}}{\left(b^{2}+a^{2}\right)\left(a^{4}+6 \varepsilon a^{2} b^{2}+b^{4}\right)}+\frac{a^{2} c_{21}}{b^{2}+3 \varepsilon a^{2}}\left(3 x^{2} y-y^{3}\right) \\
& +\frac{b^{4} c_{04}}{a^{4}+6 \varepsilon a^{2} b^{2}+b^{4}}\left(x^{4}-6 x^{2} y^{2}+y^{4}\right) .
\end{aligned}
$$

We see that the problem (2.9) to (2.11) with the elliptical boundary $S$ always has a unique polynomial solution for arbitrary values of the coefficients $a, b, c_{i j}$. If 
$S$ is a hyperbola, the problem still has the unique solution (2.13) if $b / a$ does not assume certain exceptional values. If, for instance, $a^{2}=b^{2}$, so that $S$ is a rectangular hyperbola, we must have $c_{04}=0$, but then $b_{4}$ can be arbitrary. The results for the other exceptional values of $a^{2} / b^{2}$ are similar.

Example 3. We proceed to find polynomial solutions of degree 5 of Laplace's equation (2.9) with given fifth degree boundary values

$$
\left.u(x, y)\right|_{S}=\sum_{i+j=0}^{5} b_{i j} x^{i} y^{j}
$$

on the curve

$$
S: \quad x^{4}+y^{4}=r^{4}
$$

Such a solution must be a linear combination of harmonic polynomials of degrees 0 to 5 . There are 11 such polynomials. Further, the equation (2.15) allows us to replace the terms in

$$
y^{4}, y^{5}, \text { and } x y^{4}
$$

by $r^{4}-x^{4}, y\left(r^{4}-x^{4}\right)$, and $x\left(r^{4}-x^{4}\right)$, respectively, on $S$. We are left with 18 terms in the polynomial data (2.14) and 11 coefficients in the harmonic solution. On equating coefficients, we find that the $b_{i j}$ must satisfy the following seven equations:

$$
\begin{aligned}
& b_{20}=-b_{02}, \\
& b_{30}=-\frac{1}{3} b_{12}, \\
& b_{03}=-\frac{1}{3} b_{21}, \\
& b_{40}=b_{04} \\
& b_{31}=-b_{13} \\
& b_{32}=\frac{5}{2}\left(b_{50}-b_{14}\right), \\
& b_{23}=\frac{5}{2}\left(b_{05}-b_{41}\right) .
\end{aligned}
$$

If these conditions are satisfied, our problem has the unique fifth degree polynomial solution

$$
\begin{aligned}
u=b_{00} & +\left(b_{04}+\frac{1}{6} b_{22}\right) r^{4}+\left(b_{10}+b_{14} r^{4}+\frac{1}{2} b_{32} r^{4}\right) x+\left(b_{01}+b_{05} r^{4}+\frac{1}{10} b_{23} r^{4}\right) y \\
& +b_{20}\left(x^{2}-y^{2}\right)+b_{11} x y+b_{30}\left(x^{3}-3 x y^{2}\right)+b_{03}\left(y^{3}-3 x^{2} y\right) \\
& -\frac{1}{6} b_{22}\left(x^{4}-6 x^{2} y^{2}+y^{4}\right)+b_{31}\left(x^{3} y-x y^{3}\right)-\frac{1}{10} b_{32}\left(x^{5}-10 x^{3} y^{2}+5 x y^{4}\right) \\
& -\frac{1}{10} b_{23}\left(5 x^{4} y-10 x^{2} y^{3}+y^{5}\right) .
\end{aligned}
$$




\section{A general theorem}

The above examples suggest the result:

Theorem 1. Suppose that

$$
\alpha u_{x x}+2 \gamma u_{x y}+\beta u_{y y}=0
$$

is a second order equation with constant coefficients that do not all vanish. Let $S$ be a second degree curve

$$
S: \quad P(x, y)=0
$$

where $P(x, y)$ is given by (2.6) and $A, B, H$ are not all zero. We suppose also that no solution $u$ of (3.1), which is a polynomial of degree $N$ in $x, y$ where $N \geq 2$, vanishes identically on $S$. Then, for any given polynomial $Q_{N}(x, y)$ of degree $N$ in $x, y$, there exists a unique polynomial $u$ of degree at most $N$, such that $u$ satisfies (3.1) and

$$
u=Q_{N}(x, y) \text { on } S \text {. }
$$

We consider the class $K_{N}$ of solutions $u$ of (3.1) which are polynomials in $x, y$ of degree at most $N$ and proceed by induction on $N$. If $N=1$, then $Q_{N}(x, y)$ is linear and $u=Q_{N}$ is itself the unique linear polynomial which satisfies (3.1) and (3.2).

Suppose that Theorem 1 is proved for all degrees less than $N$. Let $P$ be given by (2.6). If $A \neq 0$, we can use (2.6) to express $x^{2}$ by terms of degree less than 2 and $x y$ and $y^{2}$ on $S$. Similarly, $x^{2 m}$ can be expressed as a polynomial of degree at most $2 m$ in $x, y$, but which is linear in $x$. We proceed similarly if $B \neq 0$, expressing $Q_{N}$ as a polynomial of degree at most $N$ in $x, y$, which is linear in $y$. If $A=B=0$, but $H \neq 0$, we introduce new variables $X, Y$ by $X=x+y, Y=x-y$. We then can express $Q_{N}$ as a polynomial of degree at most $N$ in $X, Y$ but linear in $Y$.

Thus, in all cases, on $S$ we can write

$$
Q_{N}=X R_{N-1}(Y)+S_{N}(Y)
$$

where $R_{N-1}, S_{N}$ are polynomials of degree $N-1, N$, respectively, and $(X, Y)$ are $(x, y),(y, x)$ or $(x+y, x-y)$. By hypothesis, our problem has a unique solution for polynomials of degree less than $N$. So we now can subtract the corresponding solutions for the terms of degree less than $N$ in $Q_{N}$ and are left with the polynomial data

$$
Q_{N}^{\prime}=a X Y^{N-1}+b Y^{N}
$$

where $a$ and $b$ are constants and for which a corresponding solution is to be found.

We now note that, for $N \geq 1$, the equation (3.1) has 2 linearly independent and homogenous polynomial solutions of degree $N$. For the harmonic equation (2.1) with $\varepsilon=-1$, these are the real and imaginary parts of $(X+i Y)^{N}$. For the hyperbolic equation (2.1) with $\varepsilon=1$, we may take $(X+Y)^{N}$ and $(X-Y)^{N}$. Finally, for the parabolic case $u_{X X}=0$, the polynomials are precisely the polynomials (3.3). The equation (3.1) always can be reduced to one of these 3 cases by linear transformations of the independent variables. Using the equation of $S$, we now write on $S$ a combination of these linearly independent solutions in the form

$$
A X Y^{N-1}+B Y^{N-1}+\text { terms of degree less than } N
$$

in $X, Y$ where $A, B$ are arbitrary constants. Writing $a=A, b=B$, we are left with a polynomial on $S$ of degree less than $N$, which is equal on $S$ to a polynomial 
solution by the inductive hypothesis. This completes the inductive step for existence. The uniqueness also follows since otherwise, by taking a difference, we could obtain a solution of degree at most $N$ which is not identically zero, but vanishes on $S$ and this is excluded by the hypotheses of Theorem 1 . We remark that the uniqueness of the solution follows from the maximum principle in the case of an elliptic equation (3.1) and an ellipse $S$. In other cases, the additional condition may be necessary, as Example 1 shows. For more details on the question of uniqueness, see [4].

\section{Equations with linear terms}

We now consider briefly the general equation

$$
\alpha u_{x x}+2 \gamma u_{x y}+\beta u_{y y}+2 \delta u_{x}+2 \varepsilon u_{y}+\eta u=0 .
$$

If $\eta \neq 0$, there can be no polynomial solutions. For if $u$ is a polynomial of degree $N$, the partial derivatives $u_{x}, u_{y}$, etc., all have degree less than $N$, and so the lefthand side of (4.1) has degree $N$ and cannot be zero. There are other cases where no polynomial solutions are possible. For instance, the equation

$$
u_{x x}+u_{x}=0
$$

has the general solution

$$
u=g_{1} e^{-x}+g_{2}
$$

where $g_{1}, g_{2}$ are functions of $y$. Thus, the only polynomial solutions are polynomials in $y$, and we cannot obtain a solution which is a polynomial in $(x, y)$ that assumes nonconstant polynomial data on the real axis.

A more interesting case is the heat equation

$$
u_{x x}-u_{y}=0
$$

We suppose that $u$ is a polynomial

$$
u=a_{n} x^{n}+a_{n-1} x^{n-1}+\cdots+a_{0}
$$

where $a_{0}$ to $a_{n}$ are polynomials in $y$ and $a_{n} \not \equiv 0$. Then,

$$
\begin{aligned}
u_{x x} & =n(n-1) a_{n} x^{n-2}+\cdots+2 \cdot 1 a_{2}, \\
u_{y} & =x^{n} \frac{\partial a_{n}}{\partial y}+\cdots+\frac{\partial a_{0}}{\partial y} .
\end{aligned}
$$

Equating coefficients, we see that

$$
\frac{\partial a_{n}}{\partial y}=\frac{\partial a_{n-1}}{\partial y}=0
$$

and

$$
\frac{\partial a_{j}}{\partial y}=(j+2)(j+1) a_{j+2}, \quad j=0,1,2, \ldots, n-2 .
$$

This yields $a_{n}=$ constant, $a_{n-1}=$ constant,

$$
\begin{aligned}
& a_{n-2}=n(n-1) a_{n} y+b_{n-2}, \\
& a_{n-3}=(n-1)(n-2) a_{n-1} y+b_{n-3},
\end{aligned}
$$

etc. We obtain the linearly independent polynomials

$$
P_{n}(x, y)=x^{n}+n(n-1) x^{n-2} y+\frac{n(n-1)(n-2)(n-3)}{2} x^{n-4} y^{2}+\cdots \text {. }
$$


The only polynomial solutions of (4.2) are linear combinations of the $P_{n}(x)$, given by (4.3). We note that there is exactly one polynomial $P_{n}$ of degree $n$ in $x$ for each $n$, but that for $k \geq 1$, there are two linearly independent polynomials of degree $k$ in $y$ for each $m$, namely $P_{2 k}$ and $P_{2 k+1}$. We shall deduce that there exists a unique polynomial solution with given polynomial data $P(x)$ on a line: $y=m x+c$.

To see this, we assume that

$$
\sum_{0}^{N} a_{n} P_{n}(x, m x+c)=P(x)
$$

where $N$ is the degree of $P(x)$. If $P(x)=A_{0}=$ constant, we choose $a_{0}=A_{0}$. Suppose that a solution has been found for all polynomials $P(x)$ of degree less than $N$. Then if

$$
P(x)=A_{N} x^{N}+\cdots,
$$

we choose $a_{n}=A_{N}$ and choose $a_{0}$ to $a_{N-1}$ by solving the problem for

$$
P(x)-A_{N} P_{N}(x, m x+c),
$$

which is a polynomial of degree less than $N$.

We need to prove the uniqueness of our solution, and we can do this for more general curves than the line $y=m x+c$.

Theorem 2. Suppose that $f(x)$ is a polynomial of degree $N$ where $N \neq 2$. If $u$ is a polynomial solution of (4.2) which vanishes on the curve

$$
y=f(x)
$$

then $u=0$.

Suppose first that $N=0$ or 1 . We may write

$$
u(x, y)=\sum_{0}^{M} a_{n} P_{n}(x, y), \quad a_{M} \neq 0 .
$$

In this case we have, as $x \rightarrow \infty$,

$$
P_{n}(x, y) \sim x^{n}
$$

as $y$ is linear in $x, y=m x+c$. Thus, on this line, we have

$$
u(x, m x+c) \sim a_{M} x^{M} \quad \text { as } x \rightarrow \infty,
$$

so that $u(x, m x+c) \not \equiv 0$.

Suppose next that $N>2$, so that

$$
f(x)=b_{N} x^{N}+\cdots .
$$

Then, as $x \rightarrow \infty$,

$$
P_{2 n}(x, f(x)) \sim \frac{(2 n) !}{n !}\{f(x)\}^{n} \sim b_{N}^{n} \frac{(2 n) !}{n !} x^{N n} .
$$

Thus, if $M$ is even in (4.6), we have, as $x \rightarrow \infty$,

$$
u(x, f(x)) \sim a_{M} b_{N}^{M / 2} \frac{M !}{(M / 2) !} x^{M N / 2} .
$$


If $M$ is odd, we have similarly

$$
u(x, f(x)) \sim a_{M} b_{N}^{(M-1) / 2} \frac{M !}{((M-1) / 2) !} x^{1+N(M-1) / 2} .
$$

Thus, in both cases, $u(x, y) \not \equiv 0$ on the curve (4.5).

The result may break down for $N=2$. Thus, if $f(x)=-x^{2} / 2$, the polynomial

$$
P_{2}(x, y)=x^{2}+2 y
$$

vanishes on the curve (4.5).

The above results suggest that we cannot, in general, expect a unique solution $u$ of (4.2) which assumes given values on a quadratic curve $S$. If, for instance, $S$ consists of two lines which are not both parallel to the $y$-axis, then the values on one of these lines are sufficient to determine $u$.

On the other hand, $u$ is uniquely determined by its values on two lines $x=a, x=b$ where $a \neq b$. To see this, suppose that

$$
\begin{aligned}
& u(x, y)=Q_{a}(y) \quad \text { on } x=a \\
& u(x, y)=Q_{b}(y) \quad \text { on } x=b
\end{aligned}
$$

where $Q_{a}, Q_{b}$ are polynomials of degree at most $N$ in $y$. We suppose that the problem has already been solved for polynomials of degree less than $N$, and so we may confine ourselves to the case $Q_{a}(y)=c_{a} y^{N}, Q_{b}(y)=c_{b} y^{N}$. We note that

$$
\begin{gathered}
P_{2 N+1}(a, y)=\frac{(2 N+1) !}{N !} a y^{N}+\cdots, \\
P_{2 N}(a, y)=\frac{(2 N) !}{N !} y^{N}+\cdots .
\end{gathered}
$$

Since $a \neq b$, we may choose real constants $a_{2 N}, a_{2 N+1}$, so that

$$
a_{2 N} P_{2 N}(b, y)+a_{2 N+1} P_{2 N+1}(b, y)=c_{b} y^{N}+\cdots
$$

and

$$
a_{2 N} P_{2 N}(a, y)+a_{2 N+1} P_{2 N+1}(a, y)=c_{a} y^{N}+\cdots
$$

Thus, we can reduce our problem to the case when $Q_{a}$ and $Q_{b}$ have degree less than $N$.

Again, the solution is unique. If $a_{2 N}, a_{2 N+1}$ are not both zero and $c_{a}, c_{b}$ are defined by (4.7), (4.8), then $c_{a}$ and $c_{b}$ are not both zero. Thus, in this case,

$$
u(x, y)=\sum_{0}^{2 N+1} a_{n} P_{n}(x, y) \rightarrow \infty
$$

as $y \rightarrow \infty$ on at least one of the lines $x=a, b$, so that $u(x, y)$ cannot vanish identically on both of these lines. 
Example 4. Find the polynomial solution $u(x, y)$ of the equation (4.2), which on the parabola

$$
S: \quad y=x^{2}
$$

assumes the values

$$
\left.u(x, y)\right|_{S}=\sum_{i=0}^{4} c_{i} x^{i}
$$

This example illustrates the exceptional case $N=2$ of Theorem 2. However, since the parabola (4.9) is not on a zero set of (4.2), the conclusion of Theorem 2 still goes through.

Our solution will take the form

$$
u(x, y)=\sum_{n=0}^{4} a_{n} P_{n}(x, y)
$$

where the $P_{n}(x, y)$ are the polynomials (4.3). We replace $y$ by $x^{2}$ on $S$ and see that our solution must satisfy the condition

$$
\sum_{n=0}^{4} a_{n} P_{n}\left(x, x^{2}\right)=\sum_{i=0}^{4} c_{i} x^{i}
$$

or

$$
a_{0}+a_{1} x+3 a_{2} x^{2}+7 a_{3} x^{3}+25 a_{4} x^{4}=\sum_{0}^{4} c_{i} x^{i} .
$$

Equating coefficients and recalling (4.11) and (4.3), we obtain

$$
u(x, y)=c_{0}+c_{1} x+\frac{c_{2}}{3}\left(x^{2}+2 y\right)+\frac{c_{3}}{7}\left(x^{3}+6 x y\right)+\frac{c_{4}}{25}\left(x^{4}+12 x^{2} y+12 y^{2}\right) .
$$

\section{An example for the biharmonic equation}

Our method also can be applied successfully to higher order equations. We give an example which illustrates the method, though it may look a little artificial physically.

Example 5. Find polynomial solutions $u(x, y)$ of the equation

$$
\Delta^{2} u=\frac{\partial^{4} u}{\partial x^{4}}+2 \frac{\partial^{4} u}{\partial x^{2} \partial y^{2}}+\frac{\partial^{4} u}{\partial y^{2}}=b_{1} x+b_{2} y
$$

which satisfy

$$
\left.u\right|_{S_{1}}=0
$$

on the circle

$$
S_{1}: \quad x^{2}+y^{2}=1
$$

and

$$
\left.u_{x x x}\right|_{S_{2}}=c_{1} x+c_{2} y^{2}
$$

on the ellipse

$$
S_{2}: \quad \frac{(x-k)^{2}}{a^{2}}+\frac{y^{2}}{b^{2}}=1
$$


In order to solve this problem, we may introduce a general fifth-degree polynomial satisfying (5.1) in the form

$$
\begin{aligned}
u(x, y)=a_{00} & +a_{10} x+a_{01} y+a_{20} x^{2}+a_{11} x y+a_{02} y^{2} \\
& +a_{30} x^{3}+a_{21} x^{2} y+a_{12} x y^{2}+a_{03} y^{3}+a_{40}\left(x^{4}-y^{4}\right)+a_{31} x^{3} y \\
& +a_{22}\left(3 x^{2} y^{2}-y^{4}\right)+a_{13} x y^{3}+a_{50}\left(x^{5}-5 x y^{4}\right)+a_{41}\left(5 x^{4} y-y^{5}\right) \\
& +a_{32}\left(x^{3} y^{2}-x y^{4}\right)+a_{23}\left(5 x^{2} y^{3}-y^{5}\right)+\frac{b_{1}}{24} x y^{4}+\frac{b_{2}}{120} y^{5}
\end{aligned}
$$

In order to satisfy (5.2), we replace $y^{2}$ by $\left(1-x^{2}\right)$ in (5.6) and equate the coefficients of $1, x, x^{2}, x^{3}, x^{4}, x^{5}$ and of $y, x y, x^{2} y, x^{3} y$ and $x^{4} y$ to zero in the resulting polynomial. This leads to eleven equations. To satisfy (5.4), we similarly use (5.5) to replace $y^{2}$ by $b^{2}\left(1-(x-k)^{2} / a^{2}\right)$ in $u_{x x x}$ and $c_{1} x+c_{2} y^{2}$ and then equate the coefficients of $1, y, x, x y$, and $x^{2}$ to zero in the resulting equation derived from (5.4). This leads to a further five equations making a total of sixteen equations in the eighteen variables $a_{i j}$ occurring in (5.6). Thus, our solution will contain two arbitrary constants $A$ and $B$.

Alternatively, we may use the condition (5.2) by writing

$$
\begin{gathered}
u(x, y)=\left(x^{2}+y^{2}-1\right)\left(d_{0}+d_{1,0} x+d_{01} y+d_{10} x^{2}+d_{11} x y+d_{02} y^{2}\right. \\
\left.+d_{3,0} x^{3}+d_{21} x^{2} y+d_{12} x y+d_{03} y^{3}\right)
\end{gathered}
$$

proceeding as above with the conditions (5.1) and (5.4). Then, $d_{00}$ and $d_{01}$ may be chosen arbitrarily, and we obtain eight equations in the eight remaining variables $d_{i j}$ which can be solved uniquely.

By either method, we obtain the general solution

$$
\begin{aligned}
u=\frac{1}{24} & {\left[c_{1}-\frac{k b^{2}}{4 a^{2}}\left(b_{1}-8 c_{2}\right)+\frac{k b^{4}\left(b_{1}-8 c_{2}\right)}{4 a^{2}\left(5 a^{2}+b^{2}\right)}\right]\left(x^{4}-y^{4}+2 y^{2}-1\right) } \\
& -\frac{b^{2}}{48}\left(1-\frac{k^{2}}{a^{2}}\right)\left(b_{1}-8 c_{2}\right)\left(1-\frac{b^{2}}{5 a^{2}+b^{2}}\right)\left(x^{3}+x y^{2}-x\right)+\frac{b_{1}}{24} x y^{4} \\
& +\frac{b^{2}\left(b_{1}-8 c_{2}\right)}{96\left(5 a^{2}+b^{2}\right)}\left(x^{5}-5 x y^{4}+4 x y^{2}-x\right)-\frac{b_{1}}{48} x y^{2}-\frac{b_{2}}{144} y^{3} \\
& +\frac{b_{2}}{120} y^{5}+\left[\frac{b_{1}}{48}-\frac{b^{2}\left(b_{1}-8 c_{2}\right)}{48\left(5 a^{2}+b^{2}\right)}\right]\left(x^{3} y^{2}-x y^{4}\right)+\frac{b_{2}}{720}\left(5 x^{2} y^{3}-y^{5}\right) \\
& +(A+B y)\left(x^{2}+y^{2}-1\right)
\end{aligned}
$$

Acknowledgment. We are grateful to the referee for a number of suggestions which have greatly added to the clarity of our paper. The second author gratefully acknowledges support from the Society for the Protection of Science and Learning during the writing of this paper. 


\section{References}

1. M. H. A. Abadi and E. L. Ortiz, The algebraic kernel method for the numerical solution of partial differential equations, Numer. Funct. Anal. Optim. 12 (1991), 339-360.

2. M. Sh. Mikeladze, The Theory of Plate Bending, Nauka, ca. 1974. (in Russian)

3. Z. G. Shanidze, Automatic control problems that are reducible to Neumann problems, Soobshch. Akad. Nauk. Gruzin. S.S.R. 122 (1986), 33-36. (in Russian)

4. The correctness of some automatic control problems, Soobshch. Akad. Nauk. Gruzin. S.S.R. 125 (1987), 45-48. (in Russian)

Department of Mathematics, Imperial College, 180 Queen's Gate, London, SW7 2BZ, UNITED KINGDOM 